\title{
On the Construction of Certain Number Fields
}

\author{
Shin NAKANO \\ Gakushuin University \\ (Communicated by T. Mitsui)
}

\section{Introduction}

By a number field, we mean in this paper any finite extension of the field $\boldsymbol{Q}$ of rational numbers. For any natural number $n, \zeta_{n}$ means a primitive $n$-th root of unity. Let $l$ be an odd prime fixed throughout this paper.

It was proved by Yahagi [8] that there exist infinitely many number fields whose $l$-class groups are isomorphic to any given finite abelian $l$-group. Some weaker results had been obtained by Gerth [1] and Iimura [5]. The degrees of those number fields given in [1], [5] and [8] are all divisible by $l$, and the methods in these papers do not seem to yield any number fields with degree relatively prime to $l$, even if we require these fields to satisfy only a weaker condition to have the class number divisible by $l$.

On the other hand, Satgé [7] constructed infinitely many quadratic extensions of $\boldsymbol{Q}\left(\zeta_{l}+\zeta_{l}^{-1}\right)$, whose class numbers are divisible by $l$. This is a generalization of the result in Honda [4] where the case $l=3$ is treated.

In this paper, we shall give one of the ways of constructing extensions $K$ of a given number field $k$ (satisfying a few conditions given below), such that $[K: k] \mid l-1$ and the class numbers of $K$ are divisible by $l$. We shall show that there exist infinitely many such extensions $K$. In particular, we can apply this to the case $k$ is any proper subfield of $\boldsymbol{Q}\left(\zeta_{l}\right)$, and get a similar result to Satgé's. We can show namely that there exist infinitely many extensions of $k$ with degree $l-1$ over $Q$, which are independent of $\boldsymbol{Q}\left(\zeta_{l}\right)$ over $k$ and whose class numbers are divisible by $l$.

Our method is based on the following simple idea. Let $K$ be an arbitrary number field. According to the class field theory, the class number of $K$ is divisible by $l$ if and only if there exists an unramified 
cyclic extension $L / K$ of degree $l$. Furthermore, the existence of such an extension $L / K$ is equivalent to the existence of an unramified Kummer extension $L^{\prime} / K\left(\zeta_{l}\right)$ of degree $l$ such that $L^{\prime} / K$ is abelian. So, the condition that the class number of $K$ be divisible by $l$ can be discribed in terms of the ramification theory of Kummer extension (Proposition 1). Now, we shall give a certain polynomial $f(X) \in k[X]$ (see below $\S 1$ ) so that the field $K$ defined by $f(X)=0$ satisfies the condition given in Proposition 1. Finally, we shall show, using local conditions, that infinitely many $K$ 's exist.

Notations. $\boldsymbol{Z}$ denotes as usual the ring of rational integers. For an arbitrary field $K, K^{\times}$denotes its multiplicative group. If $K$ is a number field, then $\mathfrak{o}_{K}$ denotes the ring of integers of $K$. Moreover for a prime ideal $\mathfrak{s}$ of $K$ and $\alpha \in K^{\times}, \nu_{\sharp}(\alpha)$ denotes the order of $\alpha$ at $\mathfrak{\beta}$.

\section{§ 1. Preliminary propositions.}

Let $k$ be a number field such that $\zeta_{l} \notin k$. Put $k^{\prime}=k\left(\zeta_{l}\right)$ and $m=\left[k^{\prime}: k\right]$. Then $k^{\prime} / k$ is cyclic of degree $m$ and $m \mid l-1$. Assume that there exists a prime ideal $\mathfrak{l}$ of $k$ which is totally ramified in $k^{\prime}$. Note that $\mathfrak{l} \mid l$. Let $G$ be the Galois group of $k^{\prime} / k, s$ be a generator of $G$ and $g$ be a positive integer such that $\zeta_{i}=\zeta_{i}$. We fix $s$ and $g$. Then $G$ is isomorphic to a subgroup of $(\boldsymbol{Z} / l \boldsymbol{Z})^{\times}$under the map

$$
G \ni s^{i} \longmapsto g^{i} \bmod l \in(Z / l Z)^{\times}, \quad(0 \leqq i \leqq m-1) .
$$

We denote by $\omega$ the element $\sum_{i=0}^{m-1} g^{i} s^{-t}$ of the group ring $Z[G]$. Set

$$
F(X, Y)=\prod_{t \in G}\left(X-\zeta_{l}^{t} Y\right) \text {. }
$$

Then $F(X, Y) \in \mathfrak{o}_{k}[X, Y]$ and $F(X, 1)$ is the minimal polynomial of $\zeta_{l}$ over $k$. Take $h(X) \in \mathfrak{o}_{k}[X]$ which is constant or monic, $y \in \mathfrak{0}_{k}$ such that $h(0)$ and $l y$ are relatively prime, and a unit $\varepsilon$ of $\mathfrak{o}_{k}$. For these, we define a polynomial of $\mathfrak{o}_{k}[X]$

$$
f(X)=F(X, l y)-\varepsilon h(X)^{l} .
$$

Let $\theta$ be a root of $f(X)$. Put $K=k(\theta)$ and $K^{\prime}=K\left(\zeta_{l}\right)$. The above notations $\omega, h(X), y, \varepsilon, f(X), \theta, K$ and $K^{\prime}$ will be fixed throughout this paragraph. Denote by $f^{\prime}(X)$ the derivative of $f(X)$. Then we get $f(X) \equiv X^{m}-\varepsilon h(X)^{l}$ $(\bmod \mathfrak{l})$ and $f^{\prime}(X) \equiv m X^{m-1}(\bmod \mathfrak{l}) . \quad$ As $\mathfrak{I} \backslash m$ and $h(0)$ and $l y$ are relatively prime, $f(X) \bmod \mathfrak{l}$ is a separable polynomial of $\left(\mathfrak{o}_{k} / \mathfrak{l}\right)[X]$. This implies that $\mathfrak{l}$ is unramified in $K$. As $\mathfrak{l}$ is totally ramified in $k^{\prime}$, we have $K \cap k^{\prime}=k$. 
Therefore the Galois group of $K^{\prime} / K$ can be identified with $G$, as we shall do in the following. The group ring $Z[G]$ acts on $K^{\prime \times}$.

Lemma 1. Let $L^{\prime} / K^{\prime}$ be a cyclic extension of degree $l$. Then $L^{\prime}$ is abelian over $K$ if and only if $L^{\prime}=K^{\prime}(\sqrt[6]{\alpha})$ for some $\alpha \in\left(K^{\prime \times}\right)^{\omega}$.

Proof. See Long [5] $§ 1$.

The following lemma is well-known in the theory of Kummer extension (e.g. Hecke [3] §39).

LEMMA 2. $L^{\prime} / K^{\prime}$ is an unramified cyclic extension of degree $l$ if and only if $L^{\prime}=K^{\prime}(\sqrt[6]{\alpha})$ for some $\alpha \in \mathfrak{D}_{K^{\prime}}, \alpha \neq 0$, satisfying the following conditions:

(1) $\alpha \notin K^{\prime \prime}$.

(2) $\nu_{w^{\prime}}(\alpha) \equiv 0(\bmod l)$ for any prime ideal $\mathfrak{\beta}^{\prime}$ of $K^{\prime}$.

(3) $\alpha$ and $l$ are relatively prime and the congruence $X^{l} \equiv \alpha$ $\left(\bmod \left(1-\zeta_{l}\right)^{l}\right)$ is solvable in $\mathfrak{o}_{K^{\prime}}$.

The above two lemmas yield, in virtue of the class field theory as mentioned in the introduction, the following

Proposition 1. The class number of $K$ is divisible by $l$ if and only if there exists $\alpha \in \mathfrak{D}_{K^{\prime}}, \alpha \neq 0$, satisfying the conditions (1), (2) and (3) of Lemma 2 and that $\alpha \gamma^{l} \in\left(K^{\prime \times}\right)^{\omega}$ for some $\gamma \in K^{\prime \times}$.

Now, set $\beta=\theta-l y \zeta_{l}$ and $\alpha=\beta^{\omega}$. These are the elements of $\mathfrak{o}_{K^{\prime}}$.

LEMMA 3. (i) $N_{K^{\prime} / K} \beta=\varepsilon h(\theta)^{l}$, where $N_{K^{\prime} / K}$ is the norm map from $K^{\prime}$ to $K$. (ii) No prime factor $\mathfrak{S}^{\prime}$ of $\beta$ of $K^{\prime}$ divides $\beta^{t}$ for any $t \in G, t \geqslant 1$. (iii) All prime ideals $\mathfrak{\beta}$ of $K$ dividing $h(\theta)$ are decomposed completely in $K^{\prime}$.

Proof. By the definition of $F(X, Y)$, we have $N_{K^{\prime} / K} \beta=F(\theta, l y)=$ $\varepsilon h(\theta)^{2}$. To see (ii), assume $\beta \equiv \beta^{*^{i}} \equiv 0\left(\bmod \mathfrak{\beta}^{\prime}\right)$ for some $\mathfrak{\beta}^{\prime}$ and $s^{i} \neq 1$. Then $l y \zeta_{l}\left(1-\zeta_{l}^{i-1}\right) \equiv 0\left(\bmod \Re^{\prime}\right)$. Since $g^{i} \equiv 1(\bmod l)$, we have $1-\zeta_{l}^{i-1} \mid l$. Hence $l y \equiv 0\left(\bmod \mathfrak{B}^{\prime}\right)$ and $\theta \equiv 0\left(\bmod \mathfrak{P}^{\prime}\right)$. On the other hand, we have $h(\theta) \equiv 0\left(\bmod \Re^{\prime}\right)$, from (i). So we have $l y \equiv h(0) \equiv 0\left(\bmod \Re^{\prime}\right)$. This is a contradiction. (iii) is shown easily from (i) and (ii). So our lemma is proved. by $l$.

Proposition 2. If $\alpha \notin K^{\prime \prime}$ then the class number of $K$ is divisible

Proof. By Proposition 1, it is sufficient to show that $\alpha=\beta^{\omega}$ 
satisfies the conditions (2) and (3) of Lemma 2. Let $\mathfrak{F}^{\prime}$ be a prime ideal of $K^{\prime}$ and $\Re$ the prime ideal of $K$ defined by $\mathfrak{\$}=\mathfrak{\beta}^{\prime} \cap \mathfrak{o}_{K}$. By Lemma 3, we have $\nu_{k}(\beta)=0$ or $\nu_{k}(\beta)=\nu_{*}\left(N_{K^{\prime} / K} \beta\right)=\nu_{\sharp}\left(\varepsilon h(\theta)^{l}\right) \equiv 0(\bmod l)$. Therefore, we have $\nu_{w^{\prime}}(\alpha)=\sum_{i=0}^{m=1} g^{i} \nu_{w^{\prime}}(\beta)=0(\bmod l)$, for any prime ideal $\mathfrak{F}^{\prime}$ of $K^{\prime}$. So, (2) is satisfied. Next, $\beta \equiv \theta-l y\left(\bmod \left(1-\zeta_{l}\right)^{l}\right)$, as $(l)=\left(1-\zeta_{l}\right)^{l-1}$. Hence $\alpha=\beta^{\omega} \equiv \prod_{i=0}^{\pi-1}(\theta-l y)^{i}\left(\bmod \left(1-\zeta_{l}\right)^{l}\right)$. From the choice of $h(X)$ and $y$, we see easily that $\theta$ and $l$ are relatively prime, and so are also $\alpha$ and $l$. We have $\sum_{i=0}^{m-1} g^{i} \equiv 0(\bmod l)$, since $m \neq 1$. This shows that $\alpha$ satisfies (3), and the proof is completed.

Next, take a prime ideal $\mathfrak{p}$ of $k$ such that $N \mathfrak{p} \equiv 1(\bmod l)($ where $N \mathfrak{p}$ is the absolute norm of $\mathfrak{p}$ ). Then we can find $u \in \mathfrak{o}_{k}$ satisfying the congruence $F(u, 1) \equiv 0(\bmod \mathfrak{p})$. For such $u$, set

$$
\lambda_{k}=(l u)^{l-m} \prod_{i=1}^{m-1}\left(1-u^{\bar{b}^{i}-1}\right)^{\boldsymbol{o}^{i}-1},
$$

where $\bar{g}$ is a positive integer such that $\bar{g} g \equiv 1(\bmod l) . \quad \lambda_{\mathfrak{w}} \bmod \mathfrak{p}$ is uniquely determined in $\left(\mathfrak{o}_{k} / \mathfrak{p}\right)^{\times}$independing of the choice of $\bar{g}$ since $u^{l} \equiv 1$, $u \neq 1(\bmod \mathfrak{p})$.

Proposition 3. If (i) $f(X)$ is irreducible, (ii) $h(l y u) \equiv 0(\bmod \mathfrak{p})$ and (iii) $\varepsilon y^{l-m} \lambda_{*}$ is not an l-th power mod $\mathfrak{p}$, then the class number of $K$ is divisible by $l$.

Proof. By the choice of $u$, we have $F(l y u, l y) \equiv 0(\bmod \mathfrak{p})$. We first claim that there exists $x \in \mathfrak{v}_{k}$ such that $\mathfrak{p} \| F(x, l y)$ and $x \equiv l y u(\bmod \mathfrak{p})$. It is sufficient to show this in case $F(l y u, l y) \equiv 0\left(\bmod p^{2}\right)$. Set $\Phi(X)=F(X, l y)$ and take $\Psi(X) \in \mathfrak{D}_{k}[X]$ such that $\Phi(X) \Psi(X)=X^{l}-(l y)^{l}$. Then we have $\Phi^{\prime}(l y u) \Psi(l y u) \equiv l(l y u)^{l-1}(\bmod \mathfrak{p})$. Since $l y$ and $h(0)$ are relatively prime, and consequently $y \neq 0(\bmod \mathfrak{p})$, we get $\Phi^{\prime}(l y u)=0(\bmod \mathfrak{p})$. Set $x=l y u+\pi$, where $\pi \in \mathfrak{p}-\mathfrak{p}^{2}$. Then, using Taylor's formula,

$$
\Phi(x) \equiv \Phi(l y u)+\Phi^{\prime}(l y u) \pi \equiv \Phi^{\prime}(l y u) \pi \nRightarrow 0 \quad\left(\bmod \mathfrak{p}^{2}\right),
$$

and so we have $\mathfrak{p} \| F(x, l y)$ and $x \equiv l y u(\bmod \mathfrak{p})$. Now, from (ii), $\mathfrak{p} \mid f(x)$. On the other hand, we have $N_{K / k}(\theta-x)= \pm f(x)$, since $f(X)$ is irreducible. Hence $\mathfrak{p} \| N_{K / k}(\theta-x)$. So there exists a prime ideal $\mathfrak{F}_{\beta}$ of $K$ such that $N_{K / k} \mathfrak{P}=\mathfrak{p}$ and $\theta \equiv x(\bmod \mathfrak{\beta})$. Then we have $N_{K^{\prime} / x} \beta \equiv 0(\bmod \Re)$, since $N_{K^{\prime} / \mathbb{K}} \beta=\varepsilon h(\theta)^{l}$, and there exists a prime ideal $\mathfrak{P}^{\prime}$ of $K^{\prime}$ which divides $\beta$ and $\mathfrak{F}$. As $\mathfrak{P}$ is decomposed completely in $K^{\prime}$, we have $N_{K^{\prime} / k^{\prime}} \mathfrak{P}^{\prime}=\mathfrak{p}$.

Next, we see $\theta \equiv l y \zeta_{l}\left(\bmod \mathfrak{\beta}^{\prime}\right)$ since $\beta \equiv 0\left(\bmod \mathfrak{F}^{\prime}\right)$. On the other hand, $\theta \equiv x \equiv l y u(\bmod \Re)$. Therefore $u \equiv \zeta_{l}\left(\bmod \mathfrak{F}^{\prime}\right)$ and we get 


$$
\beta^{s^{-i}}=\theta-l y \zeta_{i}^{i} \equiv l y u\left(1-u^{\sigma^{i}-1}\right)\left(\bmod \mathfrak{P}^{\prime}\right), \quad(1 \leqq i \leqq m-1) .
$$

Set $\alpha^{\prime}=\alpha / h(\theta)^{l}$. Then $\alpha^{\prime} \in \mathfrak{o}_{K^{\prime}}$ and

$$
\alpha^{\prime}=\varepsilon \prod_{i=1}^{m-1} \beta^{e^{-i}\left(g^{i}-1\right)} \equiv \varepsilon \prod_{i=1}^{m-1}\left\{(l y u)^{\sigma^{i}-1}\left(1-u^{g^{i-1}}\right)^{\sigma^{i}-1}\right\} \quad\left(\bmod \mathfrak{P}^{\prime}\right) .
$$

As we have $\sum_{i=1}^{m-1}\left(g^{i}-1\right) \equiv-m(\bmod l)$, we get

$$
\alpha^{\prime} \equiv v^{l} \varepsilon(l y u)^{l-m} \prod_{i=1}^{m-1}\left(1-u^{j^{i}-1}\right)^{\rho^{i}-1} \equiv v^{l} \varepsilon y^{l-m} \lambda_{\star} \quad\left(\bmod \mathfrak{B}^{\prime}\right),
$$

for some $v \in \mathfrak{D}_{k}$ such that $\mathfrak{p} \backslash v$. Therefore, the assumption (iii) shows that $\alpha^{\prime}$ is not an $l$-th power mod $\mathfrak{P}^{\prime}$, since $N_{K^{\prime} / k} \mathfrak{F}^{\prime}=\mathfrak{p}$. Thus $\alpha^{\prime} \notin K^{\prime l}$ and $\alpha \notin K^{\prime \prime}$. Our proposition follows from this and Proposition 2.

REMARK. It is easy to see that for $\mathfrak{p}, u$ and $\varepsilon$ there exists $y \in \mathfrak{p}_{k}$ satisfying (iii) of Proposition 3.

\section{§ 2. Main theorem.}

THEOREM. Let $k$ be a number field such that $\zeta_{l} \notin k$ and assume that there exists a prime ideal of $k$ which is totally ramified in $k\left(\zeta_{l}\right)$. Set $m=\left[k\left(\zeta_{l}\right): k\right]$. Then there exist infinitely many number fields $K$ with the following properties:

(a) $K=k(\theta), \theta$ being any root of the polynomial $f(X)=F(X, l y)-z^{l}$, where $F(X, Y)$ is the polynomial of $\mathrm{o}_{k}[X, Y]$ as defined by $\left(^{*}\right)$ and $y, z$ are suitably chosen elements of $\mathfrak{b}_{k}$.

(b) The class number of $K$ is divisible by $l$.

(c) $K \cap k\left(\zeta_{l}\right)=k$.

(d) $[K: k]=m$.

Furthermore, in case $\zeta_{m} \notin k$, we may add the following condition on $K$ :

(e) $K / k$ is non-Galois.

Proof. We apply Proposition 3 with $\varepsilon=1$ and $h(X)=$ constant. Recall that $k\left(\zeta_{l}\right) / k$ is cyclic and $F(X, 1)$ is the minimal polynomial of $\zeta_{l}$ over $k$. So, there exists a prime ideal $\mathfrak{p}_{1}$ of $k$ such that $F(X, 1) \bmod \mathfrak{p}_{1}$ is irreducible in $\left(\mathfrak{o}_{k} / \mathfrak{p}_{1}\right)[X]$. Next, take a prime ideal $\mathfrak{p}_{2}$ of $k$ and $u \in \mathfrak{o}_{k}$ satisfying the congruences $N \mathfrak{p}_{2} \equiv 1(\bmod l)$ and $F(u, 1) \equiv 0\left(\bmod \mathfrak{p}_{2}\right)$. Obviously $\mathfrak{p}_{1} \rightleftharpoons \mathfrak{p}_{2}$ because $\mathfrak{p}_{1}$ is inert while $\mathfrak{p}_{2}$ is decomposed completely in $k_{k}\left(\zeta_{l}\right)$. Let $\lambda_{u}$ be defined by (\#). Take $y, z \in \mathfrak{o}_{k}$ such that
(i) $l y \equiv 1\left(\bmod \mathfrak{p}_{1}\right)$,
(ii) $y^{l-m} \lambda_{u}$ is not an $l$-th power $\bmod \mathfrak{p}_{2}$,
(iii) $z \equiv 0\left(\bmod \mathfrak{p}_{1}\right)$, 
(iv) $z \equiv 0\left(\bmod \mathfrak{p}_{2}\right)$,

(v) $l y$ and $z$ are relatively prime.

It is clear that such $y, z$ exist. Let $\theta$ be any root of $f(X)=F(X, l y)-z^{l}$ and $K=k(\theta)$. Then (c) is shown in $\S 1$. From (i) and (iii), we have $f(X) \equiv F(X, 1)\left(\bmod \mathfrak{p}_{1}\right)$. So $f(X)$ is irreducible in $\mathrm{o}_{k}[X]$ by the choice of $\mathfrak{p}_{1}$. Then, by Proposition 3, (b) and (d) are satisfied.

Next, we consider the case $\zeta_{m} \notin k$. We can find a prime ideal $\mathfrak{p}_{3}$ of $k$ which is not decomposed completely in $k\left(\zeta_{m}\right)$. We may assume that $\mathfrak{p}_{1}, \mathfrak{p}_{2}$ and $\mathfrak{p}_{3}$ are distinct and $\mathfrak{p}_{3} \times m$. Then it is easy to see that $y, z$ can be chosen so that the following additional conditions are satisfied:

(vi) $l y \equiv 0\left(\bmod \mathfrak{p}_{3}\right)$,

(vii) $z \equiv 1\left(\bmod \mathfrak{p}_{3}\right)$.

In this case, we have $f(X) \equiv X^{m}-1\left(\bmod \mathfrak{p}_{3}\right)$. Therefore $\mathfrak{p}_{3}$ has a prime divisor in $K$ with relative degree 1 . Assume that $K / k$ is Galois. Then $f(X) \bmod \mathfrak{p}_{3}$ factors into a product of distinct linear factors in $\left(\mathfrak{o}_{k} / \mathfrak{p}_{3}\right)[X]$. This shows that $\mathfrak{p}_{3}$ is decomposed completely in $k\left(\zeta_{m}\right)$, since the minimal polynomial of $\zeta_{m}$ over $k$ is the irreducible factor of $X^{m}-1$. This contradicts the choice of $\mathfrak{p}_{3}$, and (e) is satisfied.

To see that there are infinitely many choices of $K=k(\theta)$, it is sufficient to show that, for any finite set $S$ of such $K$ 's, there exists another field with properties (a)-(d) (and also (e) in case $\zeta_{m} \notin k$ ) which is not contained in $S$. Let $S=\left\{K_{1}, \cdots, K_{n}\right\}$. For each $i(1 \leqq i \leqq n)$, we can find a prime ideal $\mathfrak{Q}_{t} \times l$ of $K_{t}$ which is not decomposed completely in $K_{i}\left(\zeta_{l}\right)$. Put $\mathfrak{q}_{i}=\mathfrak{Q}_{i} \cap \mathfrak{o}_{k}$ and $\mathfrak{a}=\mathfrak{q}_{1} \cdots \mathfrak{q}_{n}$. Choose prime ideals $\mathfrak{p}_{1}, \mathfrak{p}_{2}$ (and $\mathfrak{p}_{3}$, if $\left.\zeta_{m} \notin k\right)$ as above which do not divide $a$. Then we can find $y, z$ satisfying in addition to (i)-(v) (and (vi), (vii), if $\zeta_{m} \notin k$ ) also the condition:

(viii) $z \equiv 0(\bmod \mathfrak{a})$.

Now, the field $K$ defined as above for such $y, z$ satisfies the properties (a)-(d) (and also (e) in case $\zeta_{m} \notin k$ ), and every prime ideal of $K$ lying above $\mathfrak{q}_{t}$ is decomposed completely in $K\left(\zeta_{l}\right)$ by Lemma $3(1 \leqq i \leqq n)$. Hence $K \notin . S$ and our theorem is proved.

Corollary 1. For any proper subfield $M$ of $Q\left(\zeta_{l}\right)$, there exist infinitely many number fields $K$ satisfying the following conditions:

(a) The class number of $K$ is divisible by $l$.

(b) $K \cap Q\left(\zeta_{l}\right)=M$.

(c) $[K: Q]=l-1$.

If $\left[Q\left(\zeta_{l}\right): M\right]>2$ i.e., $M \nsim Q\left(\zeta_{l}+\zeta_{l}^{-1}\right)$, we may add the following condition on $K$.

(d) $K / M$ is non-Galois (therefore $K / Q$ is also non-Galois). 
Corollary 2. For a given divisor $m \neq 1$ of $l-1$, there exist infinitely many extensions of $\boldsymbol{Q}\left(\zeta_{l}\right)$ of degree $m$ whose class numbers are divisible by $l$.

\section{References}

[1] F. Gerth III, Number fields with prescribed l-class group, Proc. Amer. Math. Soc., 49 (1975), 284-288.

[2] G. Gras, Sur les l-classes d'idéaux dans les extensions cycliques relatives de degré premier $l$, Ann. Inst. Fourier, vol. 23, no. 3 (1973), 1-48.

[ 3 ] E. HECKE, Vorlesungen über die Theorie der Algebraischen Zahlen, Chelsea, New York, 1970.

[4] T. HondA, On real quadratic fields whose class numbers are multiple of 3, J. Reine Angew. Math., 233 (1968), 101-102.

[5] K. IrmuRA, On the $l$-class group of an algebraic number field, J. Reine Angew. Math., 322 (1981), 136-144.

[6] R. Long, Steinitz classes of cyclic extensions of prime degree, J. Reine Angew. Math., 250 (1971), 87-98.

[7] P. SATGE, Corps résolubles et divisibilité de nombres de classes d'idéaux, Enseignement Math., 25 (1979), 165-188.

[8] O. YaHAGI, Construction of number fields with prescribed $l$-class groups, Tokyo J. Math., 1 (1978), 275-283.

[9] Y. Yамамото, On unramified Galois extensions of quadratic number fields, Osaka J. Math., 7 (1970), 57-76.

Present Address:

Department OF Mathematics

FaCULTY OF SCIENCE

GAKUSHUIN UNIVERSITY

Mejiro, Toshima-kU

TOKYo 171 